\title{
Identificação dos Agrotóxicos Associados ao Desenvolvimento de Linfoma Não-Hodgkin
}

\section{Pesticides Identification Associated with the Development of Non-Hodgkin's Lymphoma}

\author{
Luiza Flavia Veiga Francisco ${ }^{\mathrm{a}}$; Rogério Nunes da Silva ${ }^{\mathrm{b}}$; Jefferson Tennesse da Silva Vicente ; Lauriany da Silva Pereira
}

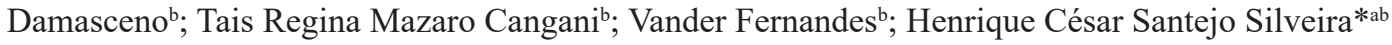

${ }^{a}$ Centro de Pesquisa em Oncologia Molecular, Hospital de Câncer de Barretos. SP, Brasil. bUniversidade de Cuiabá, Programa de Pós-Graduação Stricto Sensu em Ambiente e Saúde. MT, Brasil.

*E-mail: henriquecssilveira@gmail.com

\begin{abstract}
Resumo
A agricultura é uma das principais fontes antrópicas relacionadas com a contaminação ambiental. A quantidade de agrotóxicos utilizados nesta produção vem sendo aumentada ao longo dos anos em paralelo com o crescimento populacional. Os resíduos destes produtos contaminam o solo, o ar e a água e, com isso, apresentam risco direto ou indireto para a saúde humana e ambiental. A exposição humana aguda e crônica a estes compostos está relacionada com diversos efeitos negativos à saúde humana. O Linfoma não-Hodgkin (LNH) é um dos tipos de câncer mais associado com a exposição ocupacional aos agrotóxicos. De acordo com o levantamento de estudos realizados, neste trabalho, nos quais associaram a exposição aos agrotóxicos com LNH, 21 agrotóxicos foram identificados como associados com este tipo de câncer. Os agrotóxicos Ácido 2,4-diclorofenoxiacético, Diazinon, Diclorodifeniltricloroetano, Glifosato e Lindano foram os que apareceram em maior frequência nos artigos analisados e a associação destes compostos específicos com o risco aumentado de subtipos de LNH já foi relatada em estudos anteriores. Diante do que abordado, neste trabalho, a realização de estudos, que avaliem os riscos para o desenvolvimento de LNH causados pela exposição aos agrotóxicos se tornam necessários, a fim de contribuir com a prevenção de câncer para as populações expostas.
\end{abstract}

Palavras-chave: Agricultura. Exposição Ocupacional. Inseticidas. Herbicidas. Câncer. Toxicidade.

\begin{abstract}
Agriculture is one of the main human sources related to environmental contamination. The amount of pesticides used in this production has been increasing over the years in parallel with the increase of population. The residues of these products contaminate the soil, air and water and, therefore, present a direct or indirect risk to human and environmental health. Acute and chronic human exposure to these compounds is related to several negative effects on human health, such as the development of cancer. Non-Hodgkin Lymphoma (NHL) is one of the types of cancer most associated with occupational exposure to pesticides. According to studies collected in this work, in which they associated exposure to pesticides with NHL, 21 pesticides were identified as associated with this type of cancer. The pesticides 2,4-dichlorophenoxyacetic acid, Diazinon, Dichlorodiphenyltrichloroethane, Glyphosate and Lindane were the ones that appeared with higher frequency in the analyzed articles and the association of these specific compounds with the increased risk of NHL subtypes has been reported in previous studies. Given what was addressed in this work, studies that assess the risks to NHL development caused by exposure to pesticides are necessary in order to contribute to cancer prevention in exposed populations.
\end{abstract}

Keywords: Agriculture. Occupational Exposure. Insecticides. Herbicides. Cancer. Toxicity

\section{Introdução}

Ao longo dos últimos anos, o interesse em identificar associações entre as exposições ambientais e ocupacionais com determinados tipos de câncer vem ocorrendo de maneira crescente. Várias exposições ambientais foram propostas e investigadas como possíveis causadoras do aumento do risco de câncer. Um dos tipos de câncer, que tem sido alvo dessa investigação é o Linfoma não Hodgkin (LNH) (ALAVANJA et al., 2014; KOUTROS et al., 2019; LEON et al., 2019; TUAL et al., 2019). O LNH é um grupo heterogêneo com mais de 20 tipos diferentes de doenças causadas pela proliferação de células (linfócitos) malignas do sistema linfático, que se espalham de maneira desordenada (INCA, 2018; RIEUTORT et al., 2016). Em função do sistema linfático ser encontrado em todo o corpo, o LNH pode ser iniciado em qualquer lugar e pode ocorrer em qualquer faixa etária. Fatores como sistema imune comprometido, exposição a agentes infecciosos e produtos químicos, como os agrotóxicos têm sido associados ao seu desenvolvimento (INCA, 2018).

Para cada ano do triênio 2020-2022, de acordo com o INCA, estimam-se 12.030 novos casos de LNH, sendo 6.580 em homens e 5.450 em mulheres, assim se espera que o LNH ocupe a $8^{\circ}$ posição para os homens e $9^{\circ}$ posição para as mulheres, entre os tipos de câncer mais incidentes, em 2020, exceto pele não melanoma (INCA, 2020a). De acordo com os dados do Globocan/IARC (2018), o LNH para homens e mulheres, no Brasil, ocupou a $9^{\circ}$ posição para o tipo de câncer mais incidente e $14^{\circ}$ posição para o índice de mortalidade. Por outro lado, no Brasil, ao comparar os anos entre 1997 a 2016, foi observada diminuição no número de casos de LNH somente nos anos de 2015 e 2016, para ambos os sexos e faixa etária acima de 20 anos, porém quando analisada a taxa de mortalidade, foi observado aumento da mortalidade ao longo 
desse período (CORREA, 2019; INCA, 2020b).

A associação entre LNH e exposição a agrotóxicos têm recebido grande foco nos estudos epidemiológicos, visto que o aumento da incidência e de mortalidade, ao longo dos anos de $\mathrm{LNH}$, pode estar relacionado com o crescente aumento exponencial do uso de agrotóxicos. Estudos no Mundo todo têm demonstrado a relação do uso de agrotóxicos de diferentes classes funcionais com o aumento do risco de LNH (ALAVANJA et al., 2014; DREIHER; KORDYSH, 2006; TUAL et al., 2019).

O Brasil é o país que mais consome agrotóxicos no Mundo (INCA, 2018). Os agrotóxicos são substâncias ou mistura de substâncias químicas usados largamente em todo o Mundo na produção de diferentes culturas com a finalidade de prevenir, destruir, repelir ou mitigar qualquer ação danosa de seres vivos considerados nocivos (DAMALAS; KOUTROUBAS, 2016). Dessa maneira, os agrotóxicos são amplamente utilizados no Mundo todo em função de seus benefícios na agricultura, mantendo a alta produção de alimentos, além de serem usados na saúde pública para a redução do número de doenças transmitidas por vetores (EDDLESTON et al., 2002; ZARE et al., 2015).

Os agrotóxicos incluem diversos compostos, como inseticidas, fungicidas, herbicidas, rodenticidas, moluscicidas, nematicidas, reguladores de crescimento e outros (AKTAR; SENGUPYA; CHOWDHURY, 2009; SCHINASI; LEON, 2014). Entre esses, os herbicidas são os mais utilizados (45\%), seguidos de fungicidas (14\%) e inseticidas (12\%) (RIGOTTO et al., 2014). Apesar dos benefícios dos agrotóxicos para a agricultura se sabe que a utilização intensa destes produtos químicos se tornou um grande problema de saúde ambiental e pública, uma vez que estes compostos contaminam o ambiente (BLANKSON et al., 2016; CARVALHO, 2017; FOSU-MENSAH et al., 2016) e por estarem relacionados com o aumento do risco de toxicidade e doenças nos seres humanos (KIM; KABIR; JAHAN, 2017; MOSTAFALOU; ABDOLLAHI, 2013; PAYÁN-RENTERÍA et al., 2012).

A exposição do ser humano aos agrotóxicos pode ser ocupacional ou ambiental, no entanto, ela é mais comum em ambientes agrícolas, principalmente, entre agricultores e trabalhadores rurais, visto que as atividades que eles exercem podem incluir mistura / carregamento de produtos químicos, aplicação nos campos, limpeza de equipamentos de pulverização e controle de insetos e parasitas (APREA et al., 2016; BALDI et al., 2012; LEBAILLY et al., 2009; LEON et al., 2019). Para esse grupo de indivíduos, essa exposição pode ser perigosa, em função de seu amplo uso e não seletividade desses compostos, visto que mesmo em concentrações muito baixas, eles podem ocasionar diversos efeitos adversos à saúde humana (KIM; KABIR; JAHAN, 2017).

Entre os possíveis efeitos causados pela exposição aos agrotóxicos, resultados de vários estudos realizados no Mundo todo, têm evidenciado que indivíduos ocupacionalmente expostos aos múltiplos tipos de insumos agrícolas (agrotóxicos, fertilizantes, solventes, entre outros) constituem um grupo de maior risco para o desenvolvimento de $\mathrm{LNH}$ (ALAVANJA et al., 2014; BONNER et al., 2010; DE ROOS et al., 2003; LEON et al., 2019; LOUIS et al., 2017; PURDUE et al., 2007).

\section{Desenvolvimento}

\subsection{Metodologia}

Atualmente, o Grupo de Fatores Ambientais e Ocupacionais do Câncer do Centro de Pesquisa em Oncologia Molecular (CPOM) no Hospital de Câncer de Barretos - SP, juntamente com o Programa de Pós-Graduação Ambiente em Saúde/UNIC, realizam uma revisão sistemática, que têm como objetivo avaliar os fatores ambientais e ocupacionais envolvidos no desenvolvimento do Linfoma não Hodgkin.

O presente estudo buscou avaliar, especificamente, junto ao banco de dados da revisão sistemática, sendo realizados os artigos, que associavam a exposição aos agrotóxicos com o desenvolvimento de LNH. A estratégica de busca foi realizada até outubro de 2020, a partir de estudos indexados nos principais bancos de dados da área da saúde, como: Medline, Cochorane Library, Porta Regional de Bibliografia Virtual de Saúde - BVS, Scopus e Embase, sem restrições de idioma e ano de publicação, sendo inseridos e organizados na plataforma online Rayyan (OUZZANI et al., 2016). Foram incluídos estudos primários do tipo observacional epidemiológico do tipo caso controle, transversal e coorte. Excluíram-se trabalhos sem resumo, duplicados, sem textos completos, conferências, palestras ou eventos cartas ao editor e editoriais. Os resultados dos artigos que identificaram a associação de agrotóxicos com LNH foram separados na plataforma online Rayyan. No total foram 43 artigos com potencial associação entre a exposição aos agrotóxicos e LNH. Dessa forma, estes artigos possibilitaram o desenvolvimento deste estudo.

\subsection{Análise dos agrotóxicos no desenvolvimento de LNH}

A realização do levantamento dos estudos publicados, que associaram a exposição aos agrotóxicos com o desenvolvimento de $\mathrm{LNH}$, resultou em 21 princípios ativos associados com o risco aumentado desse tipo de câncer (ALAVANJA et al., 2014; BLAIR; CANTOR; ZAHM, 1998; BONNER et al., 2007, 2010; CHIU et al., 2004; DE ROOS et al., 2003, 2005; FISHER et al., 2020; FRITSCHI et al., 2005; HOAR et al., 1986; KACHURI et al., 2013; KOUTROS et al., 2019; LEON et al., 2019; LOUIS et al., 2017; MAHAJAN et al., 2007; MCDUFFIE et al., 2001; MILLS; YANG; RIORDAN, 2005; MOURA et al., 2020; NANNI et al., 1996, 1998; PARK et al. 2009; PURDUE et al., 2007; RUSIECKI et al., 2009; TUAL et al., 2019) conforme observado na Figura 1. 
Figura 1 - Principais agrotóxicos associados com o aumento do risco de LNH de acordo com os estudos identificados na revisão

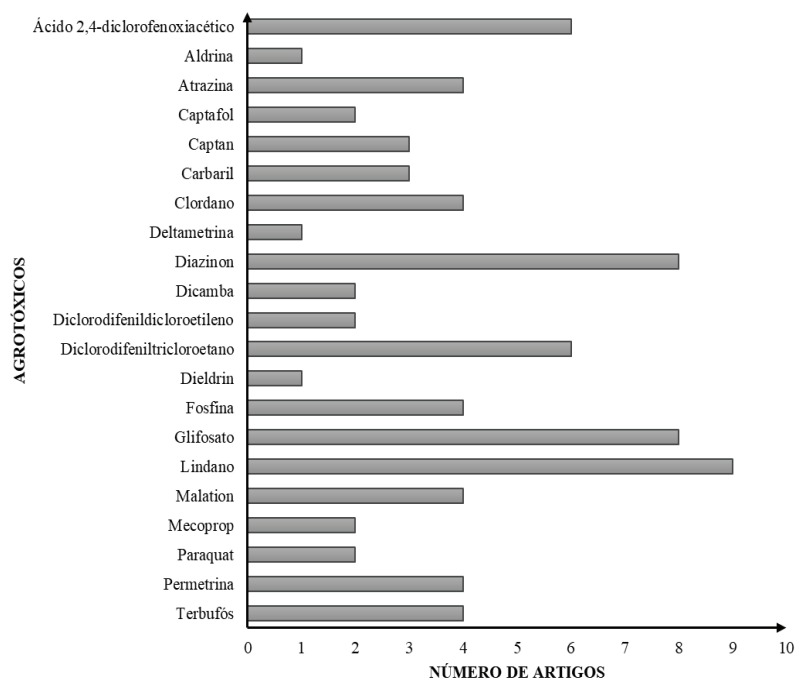

Fonte: Dados da pesquisa.

De acordo com os agrotóxicos associados com o aumento do risco de LNH, o Ácido 2,4-diclorofenoxiacético, Diazinon, Diclorodifeniltricloroetano (DDT), Glifosato e Lindano foram os que apareceram em um maior número de artigos analisados, 6, 8, 6, 8 e 9 respectivamente. Em relação ao modo de ação de todos os compostos observados, foi verificado que esses são pertencentes à diferentes classes, sendo a classe dos inseticidas com maior número de compostos associados ao LNH, conforme observado na Figura 2.

Figura 2 - Classificação dos agrotóxicos associados com o aumento do risco de $\mathrm{LNH}$, de acordo com seu modo de ação

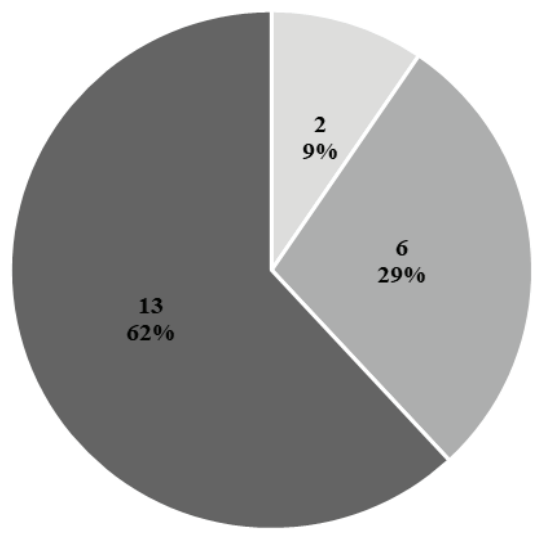

Fungicida

- Herbicida

- Inseticida

Fonte: Dados da pesquisa.

Foi também observado que dos 21 agrotóxicos identificados neste estudo com associação com o LNH, 13 apresentam classificação toxicológica pela Agência Nacional de Vigilância Sanitária (ANVISA). Os agrotóxicos Aldrin, DDT, DDE, Captafol, Lindano, Clordano e Dieldrin não apresentam classificação pela ANVISA, em função de sua utilização não ser permitida na agricultura brasileira. Para o herbicida Mecoprop, apesar de ser utilizado no Brasil, sua classificação não foi encontrada. Dessa maneira, entre os compostos identificados, neste estudo, que apresentam classificação toxicológica pela ANVISA, 5 pertencem à classe I-extremamente tóxico (Figura 3).

Figura 3 - Classificação toxicológica dos agrotóxicos associados com o aumento do risco de LNH de acordo com a ANVISA

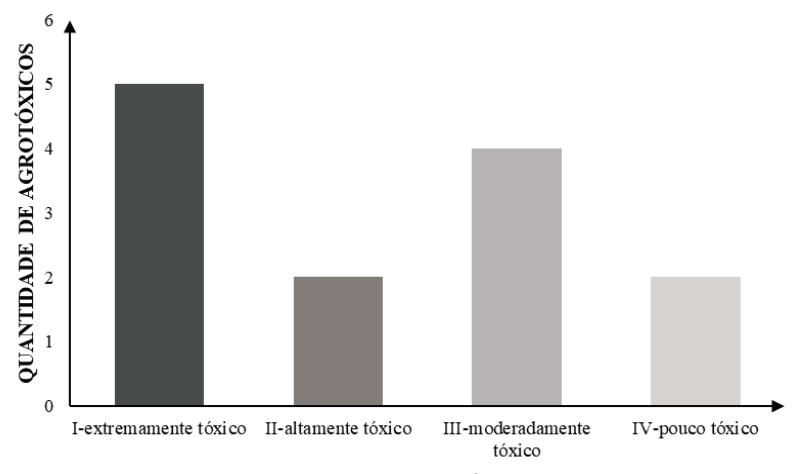

CLASSIFICAÇão TOXICOLÓGICA ANVISA

Fonte: Dados da pesquisa.

Enquanto pela classificação de carcinogenicidade realizada pela International Agency for Research on Cancer (IARC), 17 dos 21 agrotóxicos identificados neste estudo com associação ao LNH, apresentam classificação. Entre os 17 agrotóxicos, a maioria desses está no grupo 2Ae 3 ( 7 agrotóxicos em ambos os grupos), classificados como, provavelmente, carcinogênico a humanos e não carcinogênico para humanos, respectivamente (Figura 4). Para os agrotóxicos Mecoprop, Dicamba, Terbufós e Paraquat não foi encontrada a classificação pela IARC.

Figura 4 - Classificação de carcinogenicidade dos agrotóxicos associados com o aumento do risco de LNH de acordo com a IARC (International Agency for Research on Cancer).

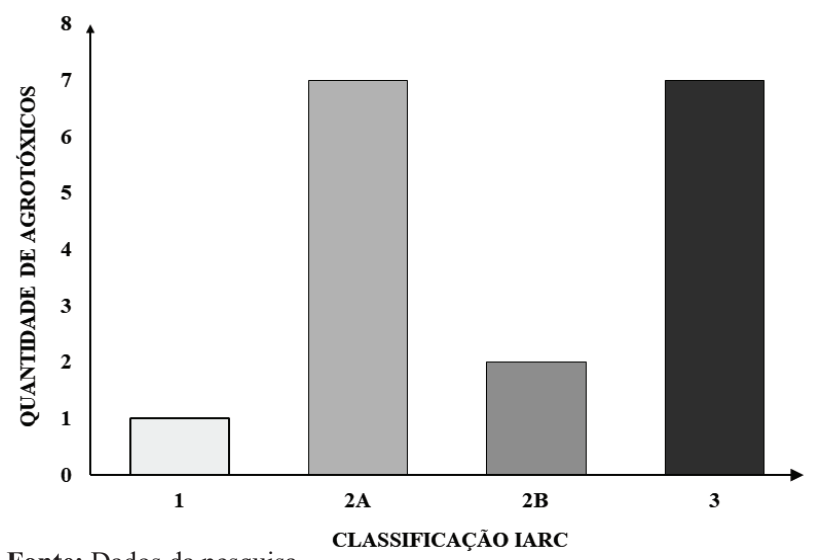

Fonte: Dados da pesquisa.

Entre os agrotóxicos que se apresentaram com maior frequência nos artigos analisados, o Lindano foi o único composto pertencente ao grupo 1 (carcinogênico a humanos) da IARC. Em estudo realizado por Tual et al. (2019), o uso de inseticidas organoclorados, como, Lindano e DDT, especialmente, em animais foi associado, positivamente, com Mieloma Múltiplo, um subtipo de LNH. Em outro estudo foi constatado que o uso constante de Lindano foi associado a riscos, significativamente, aumentados de três subtipos de 
LNH (Linfoma Folicular, Linfoma Difuso de Grandes Células B e Linfoma Linfocítico de Pequenas Células) (KACHURI et al., 2020).

Outro agrotóxico muito verificado nos estudos foi o Glifosato, esse herbicida, apesar de pertencer a classe IVpouco tóxico da ANVISA, pela classificação da IARC, esse recentemente foi classificado como, provavelmente, carcinogênico (grupo 2A) em função da ausência de estudos em humanos. Assim, como o Lindano e DDT, o Glifosato também foi associado com subtipos de LNH. De Roos et al. (2005) associaram a exposição ao Glifosato com Mieloma Múltiplo, enquanto Leon et al. (2019) o associaram com o Linfoma Difuso de Grandes Células B. O Ácido 2,4-diclorofenoxiacético foi outro herbicida muito observado entre os estudos, esse composto, diferentemente do herbicida Glifosato, pertence à classe I-extremamente tóxico pela ANVISA e 2B (possivelmente, carcinogênico para humanos) de acordo com a IARC. Apesar de suas classificações, ambos herbicidas são muito utilizados na agricultura brasileira, assim, os achados destes estudos podem ter implicações importantes na saúde pública. Em relação à associação do Ácido 2,4-diclorofenoxiacético com LNH, estudos demonstraram que a exposição a esse composto está associada com o risco aumentado de LNH geral, não sendo observada associação deste herbicida com subtipos específicos de LNH (MCDUFFIE et al., 2001; MILLS; YANG; RIORDAN, 2005).

A associação de subtipos de LNH com a exposição específica a alguns agrotóxicos foi observada por Alavanja et al. (2014), Koutros et al. (2019) e Leon et al. (2019). Terbufós e DDT foram associados ao Linfoma de Pequenas Células, Leucemia Linfocítica Crônica e Linfoma de Células Marginais, os inseticidas Lindano e Diazinon com o Linfoma Folicular, enquanto que a Permetrina com o risco aumentado de Mieloma Múltiplo (ALAVANJA et al., 2014). A associação entre Malation e os subtipos Linfoma Folicular e Linfoma Difuso de Grandes Células B, bem como a associação entre o inseticida Carbaril e Linfoma Linfocítico de Pequenas Células, também foi identificada por Koutros et al. (2019). São poucos os estudos que avaliaram o uso específico de alguns agrotóxicos para os subtipos de NHL. Porém, os artigos analisados mostram que há evidências consistentes de que a exposição a agrotóxicos, principalmente, no ambiente ocupacional pode ser um importante fator de risco para o desenvolvimento de LNH (FERRI et al., 2017).

\section{Conclusão}

O uso de agrotóxicos como fator de risco para o câncer, no contexto agrícola, tem sido amplamente estudado, nos últimos anos, como abordado anteriormente. Os efeitos negativos a saúde, associados com alguns destes produtos, podem causar grande impacto na saúde humana. Com base no levantamento realizado neste estudo, foi possível observar um total de 21 agrotóxicos associados ao desenvolvimento de LNH. De acordo com a classificação toxicológica da ANVISA, a maioria destes agrotóxicos apresenta classificação pertencente a classe I (extremamente tóxico). Por outro lado, em relação à classificação da IARC, 14 agrotóxicos dos 17 que apresentam classificação pela agência, são classificados nas categorias 2A e 3, ou seja, como provavelmente carcinogênico para humanos e não carcinogênico para humanos, respectivamente. Apesar dos estudos acima mencionados, ainda não se conhece o possível mecanismo que induz a carcinogênese causada pela exposição aos pesticidas. Assim, mais estudos que revelem os mecanismos e processos celulares são necessários para elucidar essas lacunas. Além disso, estudos epidemiológicos, que associam o aumento do risco de LNH com a exposição aos agrotóxicos, faz-se de extrema importância a realização de estudos futuros para elucidação do papel da exposição ocupacional no desenvolvimento de LNH.

\section{Referências}

AKTAR, M.W.; SENGUPTA, D.; CHOWDHURY, A. Impact of pesticides use in agriculture: their benefits and hazards. Interdisc. Toxicol., v.2, n.1, p.1-12, 2009. doi: 10.2478/v10102-009-0001-7.

ALAVANJA, M.C.R. et al. Non-Hodgkin Lymphoma Risk and Insecticide, Fungicide and Fumigant Use in the Agricultural Health Study. PLoS ONE, v.9, n.10, 2014. doi: 10.1371/journal. pone.0109332.

APREA, M C. et al. Assessment of exposure to pesticides during mixing/loading and spraying of tomatoes in the open field. J. Occup. Environ. Hyg., v.13, n.6, p.476-489, 2016. doi: 10.1080/15459624.2016.1143948.

BALDI, I. et al. Levels and determinants of pesticide exposure in operators involved in treatment of vineyards: results of the PESTEXPO Study. J. Exposure Scie. Environ. Epidemiol., v.22, n.6, p.593-600, 2012. doi: 10.1038/jes.2012.82.

BLAIR, A.; CANTOR, K.P.; ZAHM, S. H. Non-hodgkin's lymphoma and agricultural use of the insecticide lindane. Am. J. Ind. Med., v.33, n.1, p.82-87, 1998. doi: 10.1002/(sici)10970274(199801)33:1<82::aid-ajim9>3.0.co;2-y.

BLANKSON, G.K. et al. Contamination levels of organophosphorus and synthetic pyrethroid pesticides in vegetables marketed in Accra, Ghana. Food Control, v.68, p.174180, 2016. doi: .1016/j.foodcont.2016.03.045.

BONNER, M. R. et al. Malathion exposure and the incidence of cancer in the agricultural health study. Am. J. Epidemiol., v.166, n.9, p.1023-1034, 2007. doi: 10.1093/aje/kwm182.

BONNER, M.R. et al. Occupational Exposure to Terbufos and the Incidence of Cancer in the Agricultural Health Study. Cancer Causes Control : CCC, v.21, n.6, p.871-877, 2010. doi: 10.1007/ s10552-010-9514-9.

CARVALHO, F.P. Pesticides, environment, and food safety. Food Energy Security, v.6, n.2, p.48-60, 2017. doi: 10.1002/fes3.108.

CHIU, B.C.H. et al. Agricultural pesticide use, familial cancer, and risk of non-Hodgkin lymphoma. Cancer Epidemiol. Biomarkers Prevention, v.13, n.4, p.525-531, 2004.

CORREA, A.N. Observatório de Oncologia » Trajetória da incidência e mortalidade das neoplasias hematológicas no Brasil. Disponível em: https://observatoriodeoncologia.com. br/trajetoria-da-incidencia-e-mortalidade-das-neoplasiashematologicas-no-brasil/. Acesso em: 15 mar. 2020.

DAMALAS, C.A.; KOUTROUBAS, S.D. Farmers' exposure to 
pesticides: toxicity types and ways of prevention. Toxics, v. 4 , n. 1, 2016. doi: 10.3390/toxics4010001.

DE ROOS, A. J et al. Integrative assessment of multiple pesticides as risk factors for non-Hodgkin's lymphoma among men. Occup. Environ. Med., v.60, n.9, p.11, 2003. doi: 10.1136/oem.60.9.e11.

DE ROOS, A.J. et al. Cancer Incidence among GlyphosateExposed Pesticide Applicators in the Agricultural Health Study. Environ. Health Perspect., v.113, n.1, p.49-54, 2005. doi: 10.1289/ehp.7340.

DREIHER, J.; KORDYSH, E. Non-hodgkin lymphoma and pesticide exposure: 25 years of research. Acta Haematol., v. 116, n. 3, p. 153-164, 2006. doi: 10.1159/000094675.

EDDLESTON, M. et al. Pesticide poisoning in the developing world--a minimum pesticides list. Lancet, v. 360, n. 9340, p. 1163-1167, 2002. doi: 10.1016/s0140-6736(02)11204-9.

FERRI, G. M. et al. Risk of lymphoma subtypes by occupational exposure in Southern Italy. J. Occup. Med. Toxicol., v.12, 2017. doi: 10.1186/s12995-017-0177-2.

FISHER, J.A. et al. Residential Proximity to Intensive Animal Agriculture and Risk of Lymphohematopoietic Cancers in the Agricultural Health Study. Epidemiology, v.31, n.4, p.478-489, 2020. doi: 10.1097/EDE.0000000000001186.

FOSU-MENSAH, B. Y. et al. Organophosphorus pesticide residues in soils and drinking water sources from cocoa producing areas in Ghana. Environ. Syst. Res., v.5, n.1, p.10, 2016. doi: 10.1186/s40068-016-0063-4.

FRITSCHI, L. et al. Occupational exposure to pesticides and risk of non-hodgkin's lymphoma. Am. J. Epidemiol., v.162, n.9, p.849-857, 2005. doi: 10.1093/aje/kwi292.

HOAR, S.K. et al. Agricultural herbicide use and risk of lymphoma and soft-tissue sarcoma. JAMA, v.256, n.9, p.11411147, 1986.

INCA - Instituto Nacional de Câncer - Diretrizes para a vigilância do câncer relacionado ao trabalho. Rio de Janeiro: INCA, 2012.

INCA - Instituto Nacional de Câncer - Estimativa 2020 Incidência de câncer no Brasil. 2020a. Disponível em: https:// www.inca.gov.br/publicacoes/livros/estimativa-2020-incidenciade-cancer-no-brasil. Acesso em: 10 jun. 2020.

INCA - Instituto Nacional de Câncer. Atlas on-line de mortalidade. 2020b. Disponível em: https://mortalidade. inca.gov.br/MortalidadeWeb/pages/Modelo10/consultar. xhtml\#panelResultado. Acesso em: 10 jun. 2020

KACHURI, L. et al. Multiple pesticide exposures and the risk of multiple myeloma in Canadian men. Int. J. Cancer, v.133, n.8, p.1846-1858, 2013. doi: 10.1002/ijc.28191.

KACHURI, L. et al. Insecticide use and risk of non-Hodgkin lymphoma subtypes: a subset meta-analysis of the North American Pooled Project. Int. J. Cancer, 2020. doi: 10.1002/ ijc.33164.

KIM, K.-H.; KABIR, E.; JAHAN, S. A. Exposure to pesticides and the associated human health effects. Scie. Total Environ., v.575, p.525-535, 2017. doi: 10.1016/j.scitotenv.2016.09.009.

KOUTROS, S. et al. Non-Hodgkin lymphoma risk and organophosphate and carbamate insecticide use in the north American pooled project. Environ. Int., v.127, p.199-205, 2019. doi: 10.1016/j.envint.2019.03.018.

LEBAILLY, P. et al. Exposure to Pesticides in Open-field Farming in France. Ann. Occupat. Hyg., v.53, n.1, p.69-81, 2009. doi: 10.1093/annhyg/men072.
LEON, M. E. et al. Pesticide use and risk of non-Hodgkin lymphoid malignancies in agricultural cohorts from France, Norway and the USA: a pooled analysis from the AGRICOH consortium. Int. J. Epidemiol., 2019. doi: 10.1093/ije/dyz017.

LOUIS, L.M. et al. A prospective study of cancer risk among Agricultural Health Study farm spouses associated with personal use of organochlorine insecticides. Environ. Health, v.16, 2017. doi: 10.1186/s12940-017-0298-1.

LUO, D. et al. Exposure to organochlorine pesticides and nonHodgkin lymphoma: a meta-analysis of observational studies. Scie. Reports, v.6, 2016. doi: 10.1038/srep25768.

MAHAJAN, R. et al. Carbaryl exposure and incident cancer in the Agricultural Health Study. Int. J. Cancer, v. 121, n. 8, p. 17991805, 2007. doi: 10.1002/ijc.22836.

MCDUFFIE, H. H. et al. Non-Hodgkin's Lymphoma and Specific Pesticide Exposures in Men: Cross-Canada Study of Pesticides and Health. Cancer Epidemiol. Prevention Biomarkers, v.10, n. 11, p. 1155-1163, 2001.

MILLS, P. K.; YANG, R.; RIORDAN, D. Lymphohematopoietic cancers in the United Farm Workers of America (UFW), 19882001. Cancer Causes Control, v.16, n.7, p. 823-830, 2005. doi: 10.1007/s10552-005-2703-2.

MOSTAFAlOU, S.; ABDOLLAHI, M. Pesticides and human chronic diseases: Evidences, mechanisms, and perspectives. Toxicol. Appl. Pharmacol., v.268, n.2, p. 157-177, 2013. doi: 10.1016/j.taap.2013.01.025.

MOURA, L.T.R. et al. Occupational exposure to organophosphate pesticides and hematologic neoplasms: a systematic review. Rev. Bras. Epidemiol., v.23, 2020. doi: 10.1590/1980-549720200022.

NANNI, O. et al. Chronic lymphocytic leukaemias and nonHodgkin's lymphomas by histological type in farming-animal breeding workers: a population case-control study based on a priori exposure matrices. Occup. Environ. Med., v.53, n.10, p.652-657, 1996. doi: 0.1136/oem.53.10.652.

NANNI, O. et al. Multiple myeloma and work in agriculture: results of a case-control study in Forli, Italy. Cancer Causes Control: CCC, v.9, n.3, p.277-283, 1998. doi: 10.1023/a:1008821119851.

OUZZANI, M. et al. Rayyan: a web and mobile app for systematic reviews. Systematic Rev., v.5, 2016. doi: 10.1186/s13643-0160384-4.

PARK, S. K. et al. Cancer incidence among paraquat-exposed pesticide applicators in the Agricultural Health Study. Int. J. Occup. Environ. Health, v.15, n.3, p.274-281, 2009.

PAYÁN-RENTERÍA, R. et al. Effect of chronic pesticide exposure in farm workers of a Mexico community. Arch. Environ. Occup. Health, v.67, n.1, p.22-30, 2012. doi: 10.1080/19338244.2011.564230.

PURDUE, M.P. et al. Occupational exposure to organochlorine insecticides and cancer incidence in the Agricultural Health Study. Int. J. Cancer, v.120, n.3, p.642-649, 2007. doi: 10.1002/ ijc. 22258 .

RIEUTORT, D. et al. Ranking occupational contexts associated with risk of non-Hodgkin lymphoma. Am. J. Ind. Med., v.59, n.7, p.561-574, 2016. doi: 10.1002/ajim.22604.

RIGOTTO, R. M. et al. Pesticide use in Brazil and problems for public health. Cad. Saúde Pública, v.30, n.7, p.1360-1362, 2014. doi: 10.1590/0102-311XPE020714.

RUSIECKI, J.A. et al. Cancer Incidence among Pesticide Applicators Exposed to Permethrin in the Agricultural Health Study. Environ. Health Perspec., v.117, n.4, p.581-586, 2009. 
doi: 10.1289/ehp.11318.

SCHINASI, L.; LEON, M. E. Non-Hodgkin lymphoma and occupational exposure to agricultural pesticide chemical groups and active ingredients: a systematic review and meta-analysis. Int. J. Environ. Res. Public Health, v.11, n.4, p.4449-4527, 2014. doi: 10.3390/ijerph110404449.
TUAL, S. et al. Occupational exposure to pesticides and multiple myeloma in the AGRICAN cohort. Cancer Causes \& Control: $C C C$, v.30, n.11, p.1243-1250, 2019. doi: 10.1007/s10552-01901230-x.

ZARE, S. et al. The impacts of pesticides on the health of farmers in Fasa, Iran. Electr. Phys. v.7, n.4, p.1168-1173, 2015. doi: 10.14661/2015.1168-1173. 